\title{
Direct measurement of local constitutive relations, at the micrometre scale, in bulk metallic alloys
}

\author{
E. Plancher, ${ }^{\mathrm{a}, \mathrm{b}, \mathrm{c} *}$ V. Favier, ${ }^{\mathrm{a}}$ C. Maurice, ${ }^{\mathrm{b}}$ E. Bosso, ${ }^{\mathrm{c}}$ N. Rupin, ${ }^{\mathrm{c}} \mathrm{J}$. Stodolna, ${ }^{\mathrm{c}}$ D. \\ Loisnard, ${ }^{c}$ J.-B. Marijon, ${ }^{a}$ J. Petit, ${ }^{d}$ J.-S. Micha, ${ }^{e, f}{ }$ O. Robach ${ }^{\mathrm{e}, \mathrm{g}}$ and O. Castelnau ${ }^{\mathrm{a}}$

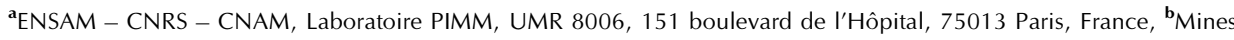 \\ Saint-Étienne, Laboratoire Georges Friedel, UMR CNRS 5307, 158 cours Fauriel, 42100 Saint-Étienne, France, 'EDF, \\ Département MMC, Avenue des renardières, 77818 Moret-sur-Loing, France, ${ }^{\mathbf{d} U n i v e r s i t e ́ ~ P a r i s ~ O u e s t, ~ L a b o r a t o i r e ~ L E M E, ~}$ \\ EA4416, 50 rue de Sèvres, 92410 Ville d'Avray, France, ${ }^{\mathbf{e} C E A-C N R S ~ C R G-I F ~ B M 32 ~ b e a m l i n e ~ a t ~ E S R F, ~} 6$ rue Jules \\ Horowitz, Cedex 9, 38043 Grenoble, France, ' Université Grenoble Alpes, INAC-SPrAM and CNRS, SPrAM and CEA, \\ INAC-SPrAM, PCI, 38000 Grenoble, France, and ${ }^{\mathrm{g}}$ Université Grenoble Alpes, INAC-SP2M and CEA, INAC-SP2M, NRS, \\ 38000 Grenoble, France. *Correspondence e-mail: emeric.plancher@mines-saint-etienne.org
}

Keywords: local constitutive behavior; stress; X-ray Laue microdiffraction; strain; digital image correlation.

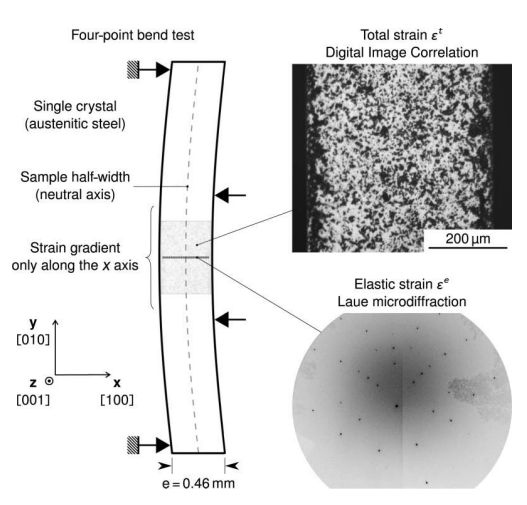

Multiscale models involving crystal plasticity are essential to predict the elastoplastic behavior of structural materials with respect to their microstructure. However, those models are often limited by a poor knowledge of the local constitutive behavior. This article reports a method to measure the mechanical behavior directly, at the micrometre scale, in bulk crystalline materials. Local strain and stress states were evaluated at the surface of a bent stainless steel crystal by combining total strain measurements - performed with the digital image correlation technique on optical images - with elastic strain measurements obtained by Laue microdiffraction. A local constitutive relation was measured, in an efficient nondestructive way, without the need for full-field simulations. The method was validated by a comparison between the measured local behavior and the macroscopic behavior of the single crystal.

\section{Introduction}

Constitutive relations based on crystal plasticity are used in full-field simulations to predict the overall mechanical response of metallic alloys. In such a multiscale approach, the local mechanical behavior of individual phases or grains in the microstructure needs to be considered (Roters et al., 2010). Therefore, in most commercial materials with a typical grain size of a few micrometres, intragranular constitutive relations must be determined with a spatial resolution of approximately $1 \mu \mathrm{m}$. At that scale, the identification of local constitutive relations is extremely challenging. Many publications suggest complex identification procedures to calibrate the constitutive law so that the actual strain field in the material can be reproduced, together with the effective behavior (Eberl, 1999; Hoc et al., 2003; Héripré et al., 2007; Suquet et al., 2012; Guery et al., 2016). Such procedures couple macroscopic loading conditions, full-field simulations of the microstructure and total strain field measurements. Good quantitative agreements between experiments and simulations have been shown on the global strain distribution in several microstructures (Tasan et al., 2014; Lim et al., 2015). However, large deviations are found locally when strain fields are compared thoroughly.

Two contradictions arise when indirect full-field identification procedures are used. First, surface strains strongly depend on the underlying microstructure. Thus, a realistic threedimensional model of the grain structure is necessary to obtain representative results. In-volume nondestructive observations 
can be achieved using three-dimensional X-ray diffraction (Poulsen, 2012), also known as HEDM (Pokharel et al., 2015) and diffraction contrast tomography (Ludwig et al., 2008; Johnson et al., 2008). Nonetheless, simplified and less accurate representations, two dimensional (Hoc et al., 2003; Guery et al., 2016) or three dimensional on a cubic lattice (Héripré et al., 2007), are usually considered in simulations to keep the computing time reasonable. Second, non-local complex constitutive equations better capture the features of experimental data such as strain gradient effects (Al-Rub \& Voyiadjis, 2004). Unfortunately, this complexity conflicts with the general requirements of successful identification procedures: an efficient model in terms of computational resources and a small set of parameters. Considering the argument above, it appears that new experimental techniques, designed to probe the local behavior in a direct manner, would unlock promising ways to calibrate constitutive laws.

Advanced characterization techniques can already provide partial data on the mechanical behavior at the micrometre scale. Nanoindentation is commonly applied to measure elastic indentation moduli. It also provides additional elastoplastic parameters, at the cost of an inverse identification procedure, based on finite element modeling (Gouldstone et $a l ., 2007)$. An alternative to nanoindentation is to test micrometre-sized samples prepared by focused ion beam (FIB) or on-chip techniques, such as pillar (Imrich et al., 2014), tensile (Kiener et al., 2008; Gravier et al., 2009) and bent (Demir et al., 2010) specimens, although great care should be taken to prevent the introduction of damage during sample preparation, such as ion implantation during FIB milling and polishing. Values of the main parameters used to characterize the slip behavior, such as the lattice friction, can be measured in that way (Monnet \& Pouchon, 2013). However, the obtained values are to be considered with caution: the behavior observed in micrometre-sized samples is often different from the bulk one because micro-samples are isolated from their bulk surroundings. Those size effects, also a concern in nanoindentation (Gouldstone et al., 2007), are avoided by the direct measurement technique suggested in the present article.

To obtain a direct measurement of the local behavior in bulk materials, data on both the total strain and the stress state must be collected simultaneously. The challenge lies in obtaining relevant components of the stress and total strain tensors for a similar volume within the crystal. Some combinations of techniques have already been explored: e.g. synchrotron X-ray diffraction (XRD) to establish relationships between local elastic strains and the macroscopic tensile load in nanocrystalline nickel (Van Swygenhoven \& Van Petegem, 2013). Other combinations include laboratory-based XRD and digital image correlation (DIC) on optical images (Chow et al., 2016), and high-angular-resolution electron backscatter diffraction and DIC on images acquired in a scanning electron microscope (SEM) (Zhang et al., 2015). However, with an arbitrary choice of techniques, adequate coupling is difficult: strains must be monitored at the same relevant scale, the volume of crystal probed must be kept constant during the measurement and absolute values of the stress tensor are needed. One successful coupling has been shown at the sub-millimetre scale: optical DIC and synchrotron XRD were used to study the yield surface of composite films under bi-axial loadings (Djaziri et al., 2013). At the micrometre scale, conventional synchrotron XRD using a monochromatic beam is hardly applicable because of the limited spatial resolution associated with high X-ray beam penetration and sample rotations required in the diffractometer.

Here, we report a direct observation of the local mechanical behavior, at the micrometre scale, in a bulk crystalline material. An experimental method has been developed to provide an efficient nondestructive way to characterize local constitutive relations. Total and elastic strain measurements were carried out to estimate both the local strain and the local stress states at the surface of a steel sample. We used Laue microdiffraction (Chung \& Ice, 1999; Robach et al., 2014) to obtain the elastic strain field and optical DIC to determine the total strain field at similar spatial resolutions. The two fields were then coupled without the need of any full-field simulation. A bent single crystal was observed in situ to validate the new method. The crystal orientation was chosen so that the only nonzero stress component was along the long $(y)$ specimen axis. In such a configuration, the local constitutive relation can be compared to the macroscopic tensile behavior of the single crystal, hence assessing the success of the method.

\section{Materials and methods}

\subsection{Overview of the in situ experiment}

Fig. 1 shows the essential features of the in situ experiment. A stainless steel single crystal, $460 \mu \mathrm{m}$ thick, was loaded in a four-point bending device. Under load, a strain gradient developed in the body of the sample along the $x$ axis. The crystal orientation was chosen so that the symmetry of the loading matches the symmetry of the crystal. In this configuration, it was shown in our previous work (Plancher et al., 2016) that a pure bending state was obtained on a similar crystal, between the central pegs of the bending device, without any noticeable out-of-axis deformation. Similar deviatoric elastic fields were observed in the present experiment (Plancher, 2015), so the stress state at each $x$ position along the $y$ axis was assumed to be purely uniaxial. The loading was interrupted five times to carry out measurements of both in-plane total strains and deviatoric elastic strains. Laue microdiffraction acquisitions were performed on profiles along the $x$ axis every $2 \mu \mathrm{m}$. Each data point is independent as the X-ray beam size was set to $0.5 \times 0.3 \mu \mathrm{m}$. Laue microdiffraction provides an absolute measurement for the deviatoric part of the elastic strain tensor, with an optimal accuracy better than $10^{-4}$ (Hofmann et al., 2011; Zhang et al., 2017). Optical images were used for DIC analysis. The contrast in the images was given by a speckle deposited on the surface of the single crystal. The total strain field was probed every $15 \mu \mathrm{m}$ to obtain surface components of the tensor, also with random uncertainties estimated to be below $10^{-4}$. The experimental 
procedure summarized here has been reported in detail by Plancher (2015).

\subsection{Sample preparation}

The austenitic stainless steel single crystal was grown by directional solidification in a horizontal furnace under argon pressure. Its chemical composition was $\mathrm{Fe}-17.0 \mathrm{Cr}-14.6 \mathrm{Ni}-$ 2.3Mo (wt \%).

The initial dislocation density in the crystal was estimated by transmission electron microscopy. One thin foil was cut by spark erosion to an initial thickness of $500 \mu \mathrm{m}$, then thinned only by electropolishing to avoid introducing new dislocations in the crystal. We used an FEI Titan microscope equipped with

(a)

Four-point bend test
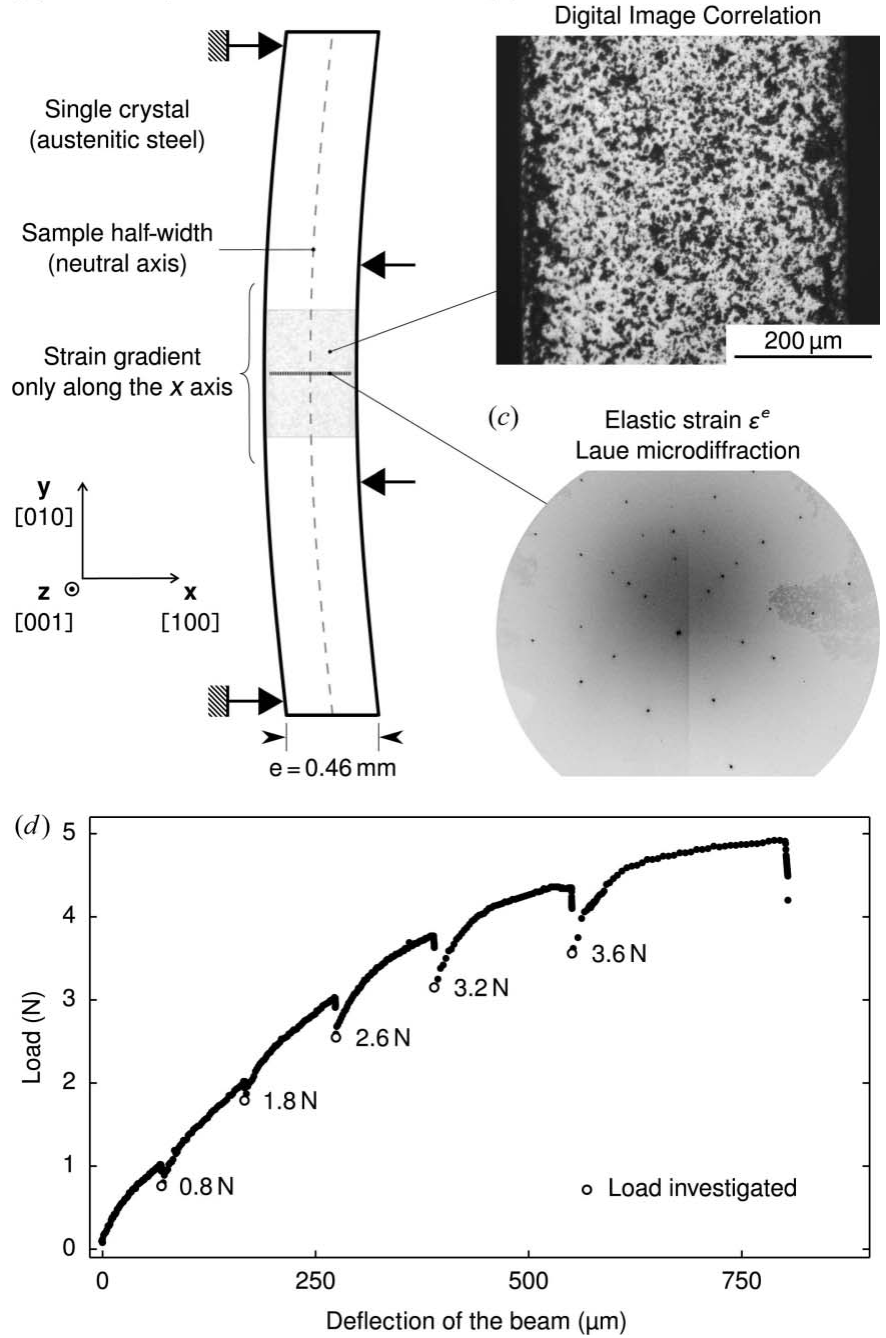

Figure 1

Characterization of the local mechanical behavior in a stainless steel single crystal. (a) Characteristics of the bend test carried out in situ at the synchrotron beamline. $(b)$ Optical image of the surface speckle used to perform digital image correlation. (c) X-ray diffraction pattern acquired for Laue microdiffraction analysis. $(d)$ Macroscopic loading curve of the interrupted bend test. The total and elastic strain fields are determined by DIC and Laue microdiffraction, respectively, when the loading is interrupted and after the force is stabilized. an energy filter to measure the foil thickness $(\sim 270 \mathrm{~nm})$ in the region of interest. A volume of $36 \mu \mathrm{m}^{3}$ was observed using conventional bright-field images. The dislocation density was calculated using the Bailey-Hirsch (Hirsch et al., 1962) formula, correcting for the diffraction condition used to acquire the images. It was found to be approximately $10^{10} \mathrm{~m}^{-2}$. As few dislocation lines were observed, this value is considered to be an upper bound for the actual dislocation density in the crystal.

The bent specimen was cut by spark erosion to an initial size of $30 \times 5 \times 0.8 \mathrm{~mm}$. The $\langle 100\rangle$ axes of the face-centered cubic (f.c.c.) crystal were aligned with the sample axes $\left( \pm 3^{\circ}\right)$ to prevent out-of-axis deformation in the bend test (Boehler \& Sawezuk, 1977). We mechanically polished the two $30 \times$ $5.0 \mathrm{~mm}$ faces with hard cloths down to a $1 \mu \mathrm{m}$ diamond grade, removing $170 \mu \mathrm{m}$ of material affected by spark erosion from each side. The observed face was also mechanically polished and then electropolished for $25 \mathrm{~s}$ (at $279 \mathrm{~K}$ under $30 \mathrm{~V}$ in Struers A2 solution). The electropolishing step was designed to eliminate any residual stress from mechanical polishing. The final size of the sample was $30 \times 4.3 \times 0.46 \mathrm{~mm}$.

The quality of the specimen preparation was checked using low-magnification channeling contrast images in a scanning electron microscope. Using optical profilometery, the surface topography was probed and found to be planar with irregularities smaller than $1 \mu \mathrm{m}$. Two subgrains were observed in the region where the strain profiles were measured. The subgrains were misoriented by 0.4 and $0.5^{\circ}$ with respect to the main crystal orientation.

A speckle of black particles was deposited on the surface of the sample for DIC analysis, using a commercial $\mathrm{MoS}_{2}$ powder (MOLYKOTE Z) with a size of 3-4 $\mu \mathrm{m}$. The contribution of the microscopic particles to the diffraction pattern was limited to few peaks of low intensity. For conventional Laue microdiffraction, the presence of these peaks was not an issue and the speckle was not disturbed by the X-ray beam.

\subsection{In situ bending experiment}

The bending experiment was performed on the microdiffraction setup available at the French CEA-CNRS beamline BM32 at the European Synchrotron Radiation Facility. A polychromatic X-ray beam with wavelengths between 5 and $23 \mathrm{keV}$ was employed. We set the beam cross section close to $0.5 \times 0.3 \mu \mathrm{m}$. Laue diffraction patterns were acquired with a MAR165 CCD detector $(2048 \times 2048$ pixels, pixel size of $79.1 \mu \mathrm{m}$, pixel depth of 16 bits). The detector was positioned vertically, $62 \mathrm{~mm}$ away from the sample. The sample was tilted $40^{\circ}$ around the $x$ axis. A similar setup was used by Hofmann $e t$ al. (2011) to estimate the uncertainty of strain measurements in a silicon single crystal, below $10^{-4}$. Similar measurement errors were also evaluated by Zhang et al. (2017) by using noisy synthetic patterns accounting for the geometric uncertainties associated with the setup calibration. A germanium single crystal was used to calibrate the diffraction geometry. Three line scans, spaced out by $100 \mu \mathrm{m}$ in the $y$ direction, were acquired with a $2 \mu \mathrm{m}$ step size along the $x$ axis. The data were 
processed with the LaueTools software (https://sourceforge. net/projects/lauetools/) using 31-33 peaks per diagram to perform the analysis. The peak intensities matched a Gaussian distribution around the peak maximum. The intensity distributions did not change in a perceptible way for total strains $\varepsilon_{y y}^{t}$ below $0.2 \%$.

The speckle images were acquired by an optical microscope with a large focal distance and low lens distortions. The field of view of the microscope was $740 \times 620 \mu \mathrm{m}$. Grayscale images were acquired with a monochromatic CCD camera, composed of $2452 \times 2054$ pixels with a depth of 8 bits. The commercial software VIC_2D (Correlated Solutions) was used to calculate the total strain field. Each image was discretized in square $100 \times 100$ pixel regions of interest (ROI), spaced on a 50 pixel grid. We used an eighth-order spline interpolation of gray levels. The displacement was calculated with a normalized correlation coefficient, searching for a homogeneous transformation of the ROI. The Lagrange strain was calculated using a smoothing of the strain field with a Gaussian filter of width 5 ROIs. Ten images acquired successively were used to estimate the static random measurement error below $7 \times$ $10^{-5}$.

The single crystal was loaded in a four-point bending setup with a distance between loading pegs of $9 \mathrm{~mm}$. The loading was incremented regularly by moving the loading pegs at a speed of $0.5 \mathrm{~mm} \mathrm{~min}^{-1}$, corresponding to a local strain rate below $2.8 \times 10^{-5} \mathrm{~s}^{-1}$. The position of the bending pegs was kept constant during the diffraction acquisitions. When the loading was interrupted, the loading force sometimes dropped owing to viscoplastic relaxation processes. Elastic and total strains were measured when the relaxation had terminated and the applied force was stabilized (about $30 \mathrm{~min}$ after the loading interruption). The beam deflection was measured by analyzing the rigid body displacement of the beam center during each loading step. The spatial positions of the elastic and total strain field measurements were controlled using the optical microscope of the microdiffraction setup. The uncertainty on the position was estimated to be below $5 \mu \mathrm{m}$.

Processing of the raw data was necessary to couple the total and elastic strain fields. First, small elastic strains (of the order of $\sim 2 \times 10^{-4}$ ) were observed at the initial state. Only strains associated with the macroscopic loading have been considered here. They were extracted from each load state by subtracting the initial elastic strain values. Second, two subgrain boundaries present in the single crystal disrupted the elastic strain field over a few micrometres. The study of this extremely local behavior would have required an observation of the total strain field with a spatial resolution of $1 \mu \mathrm{m}$. The spatial resolution of our DIC measurement $(15 \mu \mathrm{m})$ was too large for this purpose. Therefore, we chose to smooth the disruption by averaging each strain profile over three scans with positions spaced out by $100 \mu \mathrm{m}$ in the $y$ direction. Finally, we reconstructed the full elastic strain tensor from the measurement of its deviatoric part with the method detailed in Appendix $A$ [see equation (11)]. This method has been established by considering a pure uniaxial tensile state during the bend test and the anisotropic elastic constants of the crystal.

\subsection{Validation tensile experiments}

For validation purposes, two tensile samples were cut out from the raw single crystal by spark erosion. The gage area of both samples was $8.0 \times 3.0 \mathrm{~mm} \pm 0.1 \mathrm{~mm}$. The thickness of the first sample was $0.57 \pm 0.01 \mathrm{~mm}$. The thickness of the second sample was $0.47 \pm 0.02 \mathrm{~mm}$. Both the $\langle 100\rangle$ axes of the f.c.c. crystal were aligned with the sample axes $\left( \pm 3^{\circ}\right)$.

In order to measure accurately the total strains during the tensile experiments, SEM-based DIC was used. The $8.0 \times$ $3.0 \mathrm{~mm}$ gage surfaces were mechanically polished from both sides to an SiC1000 grade. The surface observed was further polished to a $1 \mu \mathrm{m}$ grade. A $400 \times 400 \mu \mathrm{m}$ gold pattern was then deposited using the method reported by Guery et al. (2013). We used a MICROMECHA Aquila tensile stage to perform the experiment in situ in a TESCAN MIRA3 SEM. The loading was carried out to obtain a strain rate of $1.2 \times$ $10^{-4} \mathrm{~s}^{-1}$. The loading was interrupted regularly to take a SEM backscatter image $(30 \mathrm{kV}, 0.5 \mathrm{nA}$, working distance $15 \mathrm{~mm})$. The images $(2048 \times 2048$ pixels, pixel depth of 8 bits, pixel size of $0.27 \mu \mathrm{m}$ ) were processed using the software VIC_2D. Each image was discretized in square $50 \times 50$ pixel ROIs, spaced on a 10 pixel grid. The DIC settings already presented in the bend test section were further used. The average value of the strain field along the loading direction is reported as the macroscopic strain.

\section{Results}

Fig. 2 shows how the largest principal tensile components of both total and elastic strain tensors evolve with the loading. Plancher et al. (2016) have reported all components of the deviatoric elastic strain tensor for a similar experiment performed on a sample taken from the same single-crystal ingot. The profiles in Fig. 2 are representative of the strain fields present in the area loaded in pure bending ( $c f$. Fig. 1). At $2.6 \mathrm{~N}$, Laue microdiffraction results show a linear and symmetric elastic strain profile $\left(\varepsilon_{y y}^{\mathrm{e}}\right)$. It is coincident with the total strain profile measured by DIC $\left(\varepsilon_{y y}^{\mathrm{t}}\right)$. At 3.2 and $3.6 \mathrm{~N}, \varepsilon_{y y}^{\mathrm{e}}$ is composed of three segments, whereas $\varepsilon_{y y}^{\mathrm{t}}$ is still linear. The elastic and total strains match close to the sample half-width. The gap between $\varepsilon_{y y}^{\mathrm{e}}$ and $\varepsilon_{y y}^{\mathrm{t}}$, observed on the right and left side of the profile, grows with the applied force.

The fields observed are characteristic of a well oriented $\langle 100\rangle$ single crystal, loaded under pure bending conditions, in the elastoplastic transition (Plancher et al., 2016). During the first loading steps $\left(0.8,1.8\right.$ and $\left.2.6 \mathrm{~N}, \varepsilon_{y y}^{\mathrm{t}}=\varepsilon_{y y}^{\mathrm{e}}\right)$, the mechanical response of the single crystal is purely elastic. At $3.2 \mathrm{~N}$, plasticity occurs on both sides of the sample, creating a gap between elastic and total strains. At $3.6 \mathrm{~N}$, the plastic regions extend towards the sample half-width. Near the sample halfwidth, elastic and total strain measurements still match as no plasticity has occurred. A linear $\varepsilon_{y y}^{\mathrm{t}}$ field is always observed, as expected in a pure bending experiment. The slight nonlinearity at both edges is correlated with the presence of two subgrains. Presumably, out-of-axis deformation can take place in these plastically deformed areas, leading to an observed loss 
of focus in the optical images. Considering a focal distance for the microscope of a few centimetres and a surface movement of a few micrometres, erroneous strains of $\sim 10^{-4}$ (estimated by the ratio of the two distances, i.e. $1 \mu \mathrm{m} / 1 \mathrm{~cm}$ ) are likely to be measured as a consequence of these out-of-plane movements.

Fig. 3 shows the local behavior at $x=-130 \mu \mathrm{m}$, on the tensile side of the crystal. For each loading step, the local stress is given by a Laue data point, whereas the local total strain is extracted from the DIC field. The points observed at 0.8, 1.8 and $2.6 \mathrm{~N}$ are aligned. The position of the points at 3.2 and 3.6 $\mathrm{N}$ reflects a change of slope in the curve. Fig. 4 shows the local behavior observed when combining every position in the strain profile and all applied loads. All the data points are dispersed around a single master curve, the dispersion being within $\pm 10 \mathrm{MPa}$. For stresses between -57 and $68 \mathrm{MPa}$, a straight segment is observed with a slope estimated by linear
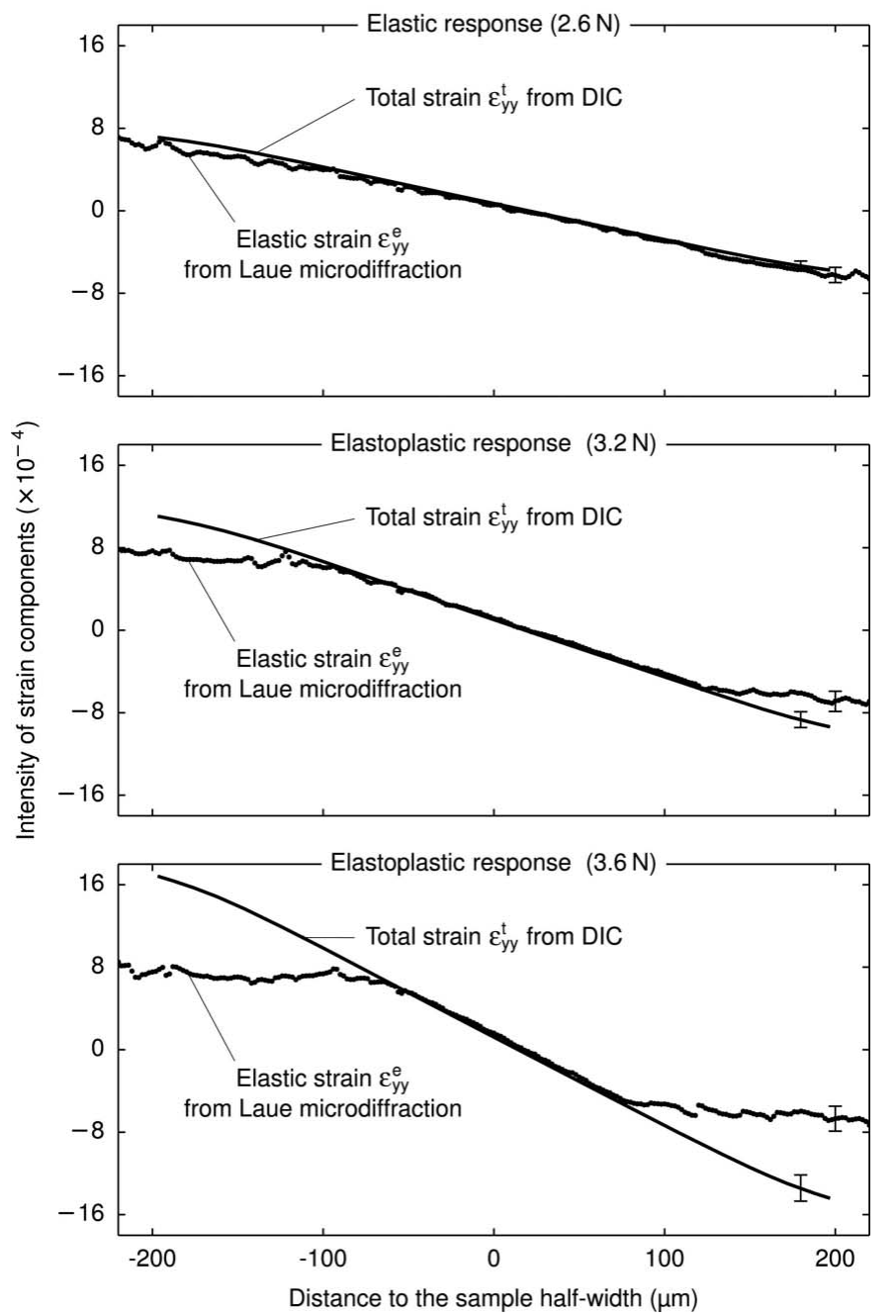

Figure 2

Evolution of total and elastic strain fields with the applied force. The largest principal strain components of the elastic $\left(\varepsilon_{y y}^{\mathrm{e}}\right)$ and total $\left(\varepsilon_{y y}^{\mathrm{t}}\right)$ strain tensors are presented. The fields are extracted from a throughthickness profile (along the $x$ direction), near the center of the beam. Error bars indicate the dispersion of the data around each mean profile with a $95 \%$ confidence interval. regression to be $98 \mathrm{GPa}$. On each side, linear segments with low slope $(\sim 8 \mathrm{GPa})$ are observed.

The measured local behavior is consistent with the expected behavior of the single crystal in the elastoplastic transition. The linear part corresponds to the elastic response of the crystal. Young's modulus measured along the [010] direction ( $98 \mathrm{GPa}$ ) is found to be close to the theoretical value of $102 \mathrm{GPa}$, computed with equation (5) from Appendix $A$ and using $C_{11}=206 \mathrm{GPa}$ and $C_{12}=133 \mathrm{GPa}$ (Ledbetter, 1981). This agreement is a direct consequence of the good match between DIC and Laue microdiffraction measurements in the

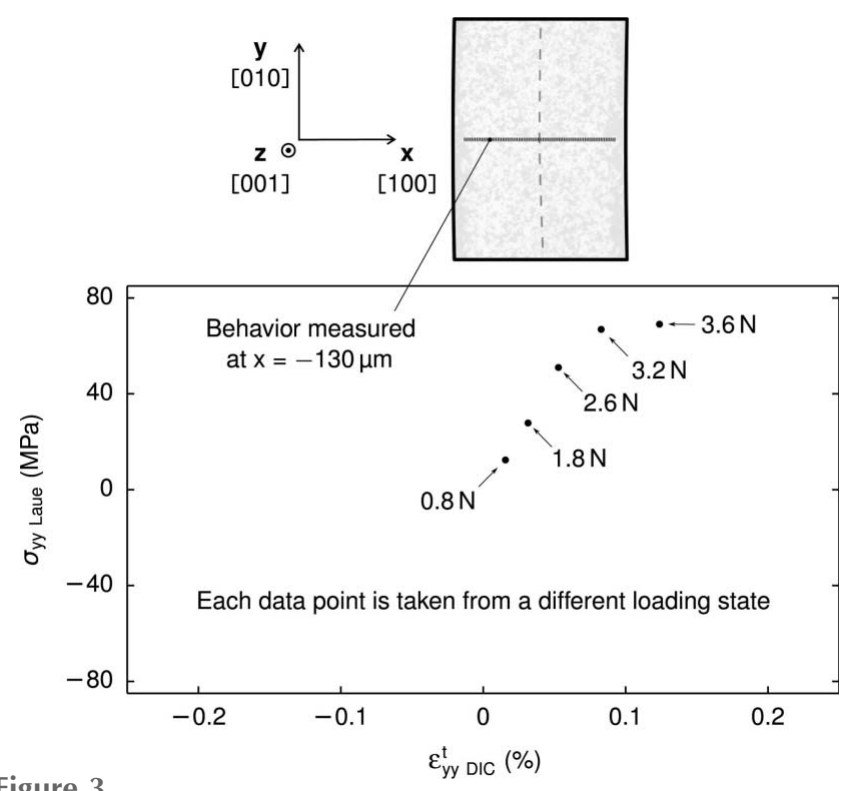

Figure 3

Direct observation of the local mechanical behavior at a specific position on the single crystal. The stress is extracted from the Laue microdiffraction data. The total strain is given by the DIC measurement.

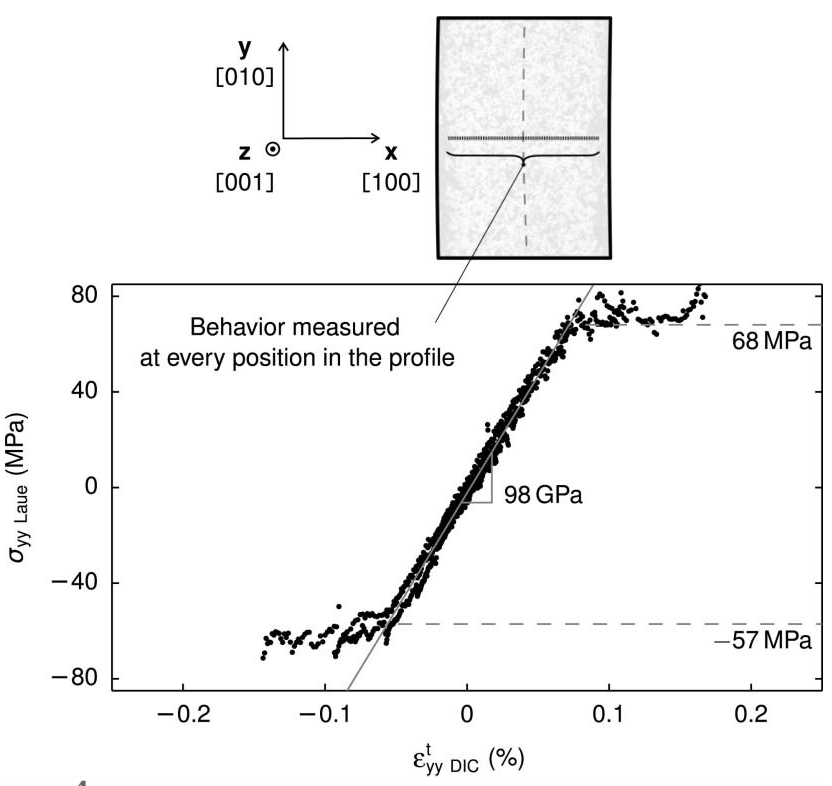

Figure 4

Direct observation of the local mechanical behavior at every point in the profile and all applied loads. The stress is extracted from the Laue microdiffraction data. The total strain is given by the DIC measurement. 
elastic regime. On both the compression and tensile sides, a clear elastoplastic transition is observed, followed by the beginning of hardening curves with a hardening modulus of $\sim 8 \mathrm{GPa}$. A dissymmetry of the behavior is observed: the yield stress in compression is $10 \mathrm{MPa}$ lower than the yield stress in tension. As discussed below, the dissymmetry probably highlights an artifact in the measurements rather than a true material characteristic. The yield stress for a symmetric behavior, taken as the mean value of the two dissymmetric values, is found to be equal to $63 \mathrm{MPa}$.

\section{Discussion}

A local stress-strain relation, at the micrometre scale, has been directly measured in a bulk material for the first time. The constitutive relation was obtained without the need of any mechanical model or identification procedure. Each micrometre-sized pixel on the specimen could be considered as an independent mechanical test. From that perspective, specific boundary conditions for each of those tests are guided by the local stress and strain fields, developing in the macroscopic specimen as deformation proceeds.

The local behavior dataset provides a direct evaluation of the critical resolved shear stress (CRSS) at which the crystal starts flowing plastically. Generally, identification of the CRSS from the mechanical response of polycrystals remains a key issue. Large dispersions for the CRSS are often found in the literature, depending on which crystal-polycrystal scale transition modeling is used: for similar $316 \mathrm{~L}$ stainless steels, Guilhem et al. (2010) used an initial value of $40 \mathrm{MPa}$, whereas El Shawish \& Cizelj (2016) used 85.2 MPa. In contrast, the direct and nondestructive local observation proposed here provides a simple estimation of the CRSS. As the single crystal has been deformed under uniaxial stress along [010], according to Schmid's law, the active slip systems are the eight systems with Burgers vectors aligned with either [110] or [011], on planes $\{111\}$. Accounting for the Schmid factor $1 / 6^{1 / 2}$, the corresponding CRSS is found to be $26 \pm 2 \mathrm{MPa}$. Since the initial dislocation density in the crystal was below $\sim 10^{10} \mathrm{~m}^{-2}$, the forest strength contribution to the CRSS is expected to be small. Therefore, the alloy lattice friction is considered to be the main contribution to the CRSS in the single crystal. This is consistent with the results reported by Monnet \& Pouchon (2013).

To further validate the results obtained in Fig. 4, we performed macroscopic tensile tests on single crystals with characteristic lengths of a few millimetres, with a strain rate of $1.2 \times 10^{-4} \mathrm{~s}^{-1}$. The tests were designed to obtain a local stress state similar to that during bending, i.e. a uniaxial stress along the [010] crystal axis. As shown in Fig. 5, the local behavior obtained by coupling elastic and total strain at the micrometre scale is in excellent agreement with the measured macroscopic behavior in terms of values and dispersion of the data. The overestimation of the stress by a few MPa seems associated with the dissymmetry of the behavior noted in Fig. 4.

The dissymmetry in Fig. 4 stems from multiple factors, including the uncertainty associated with the calibration of the diffraction instrument and the presence of initial residual elastic strains. (i) Errors in the determination of calibration parameters introduce systematic errors in the measurement of elastic strains (Zhang et al., 2017). In this experiment, a $\sigma_{y y}$ stress of $5 \mathrm{MPa}$ is correlated with a deviatoric strain $\varepsilon_{y y}^{\mathrm{e} *}$ of the order of $\sim 5 \times 10^{-5}$. As current calibration procedures lead to systematic errors of that order of magnitude (Hofmann et al., 2011), the dissymmetry in Fig. 4 is likely to be associated with the intrinsic uncertainty of the calibration procedure. (ii) In the initial state, a heterogeneous elastic strain field was observed in the sample. Compared to our previous experiment (Plancher et al., 2016), this residual field had a larger amplitude $\left(\sim 2 \times 10^{-4}\right)$ and its shape correlated with the presence of the two subgrains. Presumably, this is because the sample has been cut close to the side of the single-crystal ingot, where thermal gradients are more likely to create residual stresses. In the present analysis of the data, the initial state has been subtracted from each loading step, to observe the effect of the loading on the material response. In that sense, the material is considered homogenous and starting from a fresh stress-free state. This first approximation holds when the elastic behavior alone is involved. In the elastoplastic regime, it introduces an over- or underestimation of the total strain (up to $2 \times 10^{-4}$ ) and stress (up to $12 \mathrm{MPa}$, with a mean value of $4 \mathrm{MPa}$ ) at each position in the sample. As a result, part of the systematic shift in the yield limit could be attributed to the initial residual strain field. The order of magnitude of the initial stress values also matches the amplitude of the random noise observed in the plastic parts of Fig. 4. In future analyses, considering the initial state as a pre-strain would probably decrease the dispersion of the behavior observed.

Spatial mismatch between the elastic and total strain measurements mildly influences the behavior in Fig. 4 and would not change the value of the yield limit. From Fig. 2, we can see that the order of magnitude for the strain gradients (when plasticity occurs) is greater than $0.5 \times 10^{-5} \mu \mathrm{m}^{-1}$. For a spatial mismatch of $5 \mu \mathrm{m}$, a shift in total strain of $2.5 \times 10^{-5}$ could have been registered, a value lower than the expected accuracy of the measurement $\left(10^{-4}\right)$.

In our particular case of a single crystal loaded in bending, one loading step was in fact enough to retrieve a large part of

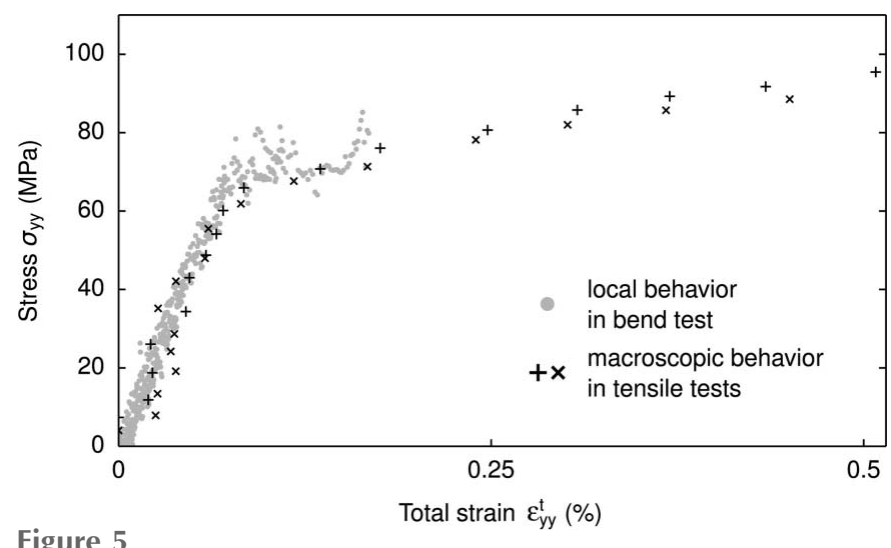

Figure 5

Local and macroscopic behavior of the single crystal loaded in uniaxial tension along the [100] direction. 
the local elastoplastic behavior. At each loading step a range of local stress state was observed along the profile. For example, the data acquired only at the $3.6 \mathrm{~N}$ load already provide a good estimation of the behavior presented in Fig. 4.

Despite the quality of those results, there is still room for further improvements. In the general case, the full elastic strain tensor is required to characterize the constitutive relation, although only the deviatoric strain was measured here. Energy-resolved Laue measurements allow estimation of the hydrostatic part of the strain tensor (Robach et al., 2011, 2013): such measurements provide the volume of the crystal unit cell. However, a simpler hypothesis of uniaxial loading could be used in this work, to retrieve the full elastic strain tensor from the measurement of its deviatoric part. The justification for this assumption has been discussed in a previous publication (Plancher et al., 2016), where similar deviatoric elastic strain fields were observed. It was shown that (i) no significant shear was detected, (ii) the normal components of the deviatoric elastic fields were consistent with a Poisson effect associated with a uniaxial stress state and (iii) an analytical model based on Bernoulli's assumption of pure bending (i.e. uniaxial stress state) describes accurately the evolution of the deviatoric strain components with the loading. This assumption of uniaxial loading was helpful to measure the elastic behavior. However, it is not needed for studying the plastic behavior. Indeed, plasticity is entirely driven by the deviatoric part of the stress tensor and, in materials with a cubic crystal lattice as here, the deviatoric stress can be determined from the sole knowledge of the deviatoric strain (Petit et al., 2015).

Small deformation levels $(<0.2 \%)$ are observed here but the experimental techniques have not reached their limits. The coupling method is expected to work for higher deformation levels, probably up to a few percent of deformation with the current procedure. Therefore, it would provide a better observation of the hardening behavior. The first limitation expected is a larger uncertainty associated with elastic strain measurements when the plastic deformation increases. Indeed, when significant plasticity is involved, strain and orientation gradients distort the shape of diffraction peaks, leading to a higher uncertainty in the detection of their position. A route suggested to deal with those plasticity-related artifacts is the Laue-DIC technique (Petit et al., 2015; Zhang et al., 2015). This technique, which uses DIC to evaluate the displacement of diffraction peaks, is slightly sensitive to an evolution of their shapes. Used in conjunction with conventional Laue microdiffraction, it is a promising way to obtain reliable results in gently deformed (poly)crystals. As plasticity develops in the sample, surface roughening and out-of-plane motions induce errors in two-dimensional DIC analyses. The use of a stereovision system (e.g. three-dimensional DIC) could solve this issue (Sutton et al., 2008), without inducing major changes in the procedure. The coupling of total and elastic strain measurements is expected to work also with other techniques such as Kossel (Bouscaud et al., 2014) and SEM-based DIC (Sutton et al., 2007).
In the present work, the spatial resolution of the DIC measurement is $15 \mu \mathrm{m}$. This was sufficient to achieve a good analysis of the smooth total strain field, at the micrometre scale, in a homogeneous single crystal. To study polycrystalline microstructures with fine grains and to observe the behavior near interfaces, the current method has to be improved in two directions. First, the spatial resolution of the DIC measurements must be improved, for example (i) by using a finer $\mathrm{MoS}_{2}$ powder to produce a refined speckle in optical images or (ii) by using atomic force microscopy or SEM images. Second, the in-depth spatial resolution of the Laue microdiffraction measurement must be improved. When plastic deformation occurs, strain and rotation gradients are expected to develop under the specimen surface. Conventional Laue microdiffraction measurements, with a penetration depth along the beam direction of $\sim 40 \mu \mathrm{m}$, would not be comparable with the DIC surface measurement anymore. For such cases, the depth profiling technique, proposed by Larson et al. (2002) and Levine et al. (2006) and extensively tested by Marijon (2017), can be used to yield the elastic strain field with a (sub)micrometric in-depth spatial resolution. This technique is available at beamline BM32 at the European Synchrotron Radiation Facility. In the present work, the strain fields observed in the well oriented single crystal are constant in the $y z$ plane. The strain state is constant along the penetration of the beam, and therefore a depth-resolved measurement was unnecessary.

As mentioned above, the continuous loading had to be interrupted multiple times to perform XRD acquisitions, as the acquisition frequency of the MARCCD detector is approximately $0.1 \mathrm{~Hz}$. This leads to a relaxation of the specimen, observed in Fig. 1. The strain rate during the bend test is also highly heterogeneous within the specimen, with a maximum value of $0.28 \times 10^{-4} \mathrm{~s}^{-1}$. Therefore, the excellent agreement between the local stress-strain responses from the interrupted bend test and the continuous tensile test could be surprising. In the bend test, the response is measured for a relaxed state, whereas in the tensile test, the response reflects the prescribed strain rate. However, strain-rate sensitivity in stainless steel is rather small. Generally, relaxation is a common problem for most in situ stress measurements (Dakhlaoui et al., 2006) and the stress relaxation phenomenon is not completely understood. Recently, a dislocation-based constitutive model, accounting for statistical distributions of internal stresses, has been proposed in the context of elastovisco-plastic polycrystalline material (Wang et al., 2016). Deeper investigation of this issue will require specific experiments with faster X-ray detectors. In the near future, a $10 \mathrm{~Hz}$ detector will be available on beamline BM32.

At the submicrometre and nano scales, similar direct observations of constitutive relations are to be reached soon, given the development of sub-micrometre beams generated by various X-ray optical systems (Mimura et al., 2010), and given the application of DIC techniques to images produced by high-spatial-resolution microscopes such as atomic force (Knauss et al., 2003) and scanning tunneling microscopes (Vendroux \& Knauss, 1998a,b). 
Direct measurements of the local behavior inherently capture size and interface effects. The knowledge of the crystal behavior in critical spots of the microstructure will improve lifetime predictions of metallic structures, a topic of major concern for many industries.

\section{Conclusions}

A direct measurement of the local constitutive relation has been performed, at the micrometre scale, on a bulk metallic alloy. Laue microdiffraction and optical DIC were used to monitor the stress and total strain fields with a micrometre spatial resolution, in a single crystal bent in the elastoplastic regime. The strains could be measured to an accuracy of the order of $10^{-4}$ (for total strains $<0.2 \%$ ) and their coupling led to the determination of the local behavior with an uncertainty of $\sim 10 \mathrm{MPa}$. The CRSS of the 316-type austenitic stainless steel crystal was evaluated as $26 \pm 2 \mathrm{MPa}$. The present technique has been validated by comparing the local stress-strain relation with that obtained via a macroscopic tensile test on the same material. We have shown that it provides an efficient nondestructive way to identify local constitutive relations, without the need for full-field simulations.

\section{APPENDIX $A$}

This section introduces the relation used to reconstruct the full elastic strain tensor, $\boldsymbol{\varepsilon}^{\mathrm{e}}$, from the measurement of its deviatoric part, $\boldsymbol{\varepsilon}^{\boldsymbol{e}^{*}}$, assuming a uniaxial stress state along the $y$ axis of the sample.

The stress tensor $\boldsymbol{\sigma}$ thus reads

$$
\boldsymbol{\sigma}=\sigma_{y y} \mathbf{y} \otimes \mathbf{y},
$$

where $\mathbf{y}$ is a unit vector of the coordinate system established in Fig. 1. The trace of the stress tensor is $\operatorname{trace}(\sigma)=\sigma_{y y}$. The deviatoric part of $\sigma$ reads

$$
\boldsymbol{\sigma}^{*}=\frac{2}{3} \sigma_{y y} \mathbf{y} \otimes \mathbf{y}-\frac{1}{3} \sigma_{y y}(\mathbf{x} \otimes \mathbf{x}+\mathbf{z} \otimes \mathbf{z}) .
$$

In the experiment, the orientation of the single crystal was chosen so that the coordinate system $(\mathbf{x}, \mathbf{y}, \mathbf{z})$ coincides with the crystal axes. Taking into account the cubic symmetry of the f.c.c. crystal, the elastic constitutive equations $\boldsymbol{\varepsilon}^{\mathrm{e}}=\mathbf{S}: \boldsymbol{\sigma}$ and $\boldsymbol{\sigma}=\mathbf{C}: \boldsymbol{\varepsilon}^{\mathrm{e}}$ are written using Voigt notation as

$$
\left[\begin{array}{c}
\varepsilon_{x x}^{\mathrm{e}} \\
\varepsilon_{y y}^{\mathrm{e}} \\
\varepsilon_{z z}^{\mathrm{e}}
\end{array}\right]=\frac{1}{E_{[100]}}\left[\begin{array}{ccc}
1 & -v & -v \\
-v & 1 & -v \\
-v & -v & 1
\end{array}\right]\left[\begin{array}{l}
\sigma_{x x} \\
\sigma_{y y} \\
\sigma_{z z}
\end{array}\right]
$$

and

$$
\left[\begin{array}{c}
\sigma_{x x} \\
\sigma_{y y} \\
\sigma_{z z}
\end{array}\right]=\left[\begin{array}{lll}
C_{11} & C_{12} & C_{12} \\
C_{12} & C_{11} & C_{12} \\
C_{12} & C_{12} & C_{11}
\end{array}\right]\left[\begin{array}{c}
\varepsilon_{x x}^{\mathrm{e}} \\
\varepsilon_{y y}^{\mathrm{e}} \\
\varepsilon_{z z}^{\mathrm{e}}
\end{array}\right] .
$$

In the relations above, only nonzero stress and strain components are shown. $E_{[100]}$ is the Young modulus along the [100] crystallographic direction and $v$ is the associated Poisson ratio. These two variables are linked with the elastic constants $C_{11}$ and $C_{12}$ according to

$$
E_{[100]}=C_{11}\left(\frac{1-3 v^{2}-2 v^{3}}{1-v^{2}}\right) \quad \text { and } \quad v=\frac{1}{1+C_{11} / C_{12}} .
$$

To establish a link between $\boldsymbol{\varepsilon}^{\mathrm{e}}$ and $\boldsymbol{\varepsilon}^{\mathrm{e} *}$, the relation $\boldsymbol{\varepsilon}^{\mathrm{e}}=\mathbf{S}: \boldsymbol{\sigma}$ is developed by introducing the deviatoric part of $\boldsymbol{\sigma}$ :

$$
\boldsymbol{\varepsilon}^{\mathrm{e}}=\mathbf{S}:\left[\boldsymbol{\sigma}^{*}+\frac{1}{3} \operatorname{trace}(\boldsymbol{\sigma}) \mathbf{I}\right] .
$$

In crystals with cubic symmetry, the stiffness and compliance tensors can be used to relate deviatoric stress and strain tensors, e.g. $\boldsymbol{\varepsilon}^{\mathrm{e} *}=\mathbf{S}: \boldsymbol{\sigma}^{*}$ (Petit et al., 2015), and therefore equation (6) becomes

$$
\boldsymbol{\varepsilon}^{\mathrm{e}}=\mathbf{S}: \boldsymbol{\sigma}^{*}+\frac{1}{3} \operatorname{trace}(\boldsymbol{\sigma}) \mathbf{S}: \mathbf{I}=\boldsymbol{\varepsilon}^{\mathrm{e} *}+\frac{1}{3} \operatorname{trace}(\boldsymbol{\sigma}) \mathbf{S}: \mathbf{I} .
$$

According to equation (2),

$$
\operatorname{trace}(\boldsymbol{\sigma})=\sigma_{y y}=\frac{3}{2} \sigma_{y y}^{*} .
$$

Given that relation (4) holds between deviatoric stress and strain,

$$
\operatorname{trace}(\boldsymbol{\sigma})=\frac{3}{2}\left[C_{11} \varepsilon_{y y}^{\mathrm{e} *}+C_{12}\left(\varepsilon_{y y}^{\mathrm{e} *}+\varepsilon_{z z}^{\mathrm{e} *}\right)\right],
$$

and with equations (3) and (5), one has

$$
\mathbf{S}: \mathbf{I}=\frac{1-2 v}{E_{[100]}} \mathbf{I}=\frac{1}{\left(C_{11}+2 C_{12}\right)} \mathbf{I} .
$$

Finally, considering equations (9) and (10) to substitute for the relevant terms in equation (7), one obtains

$$
\boldsymbol{\varepsilon}^{\mathrm{e}}=\boldsymbol{\varepsilon}^{\mathrm{e} *}+\left[\frac{C_{11}}{2\left(C_{11}+2 C_{12}\right)} \varepsilon_{y y}^{\mathrm{e} *}+\frac{C_{12}}{2\left(C_{11}+2 C_{12}\right)}\left(\varepsilon_{y y}^{\mathrm{e} *}+\varepsilon_{z z}^{\mathrm{e} *}\right)\right] \mathbf{I} .
$$

With equation (11), the full elastic strain tensor is calculated from its deviatoric part, given that a uniaxial stress state is present in the sample.

\section{Acknowledgements}

The Laboratoire de Mécanique des Solides (LMS) kindly provided the optical microscope. We acknowledge the effort of J. Monnatte and this team for building the functional microbending device. We thank O. Geaymond and O. Ulrich for their help with setting up the Laue microdiffraction instrument. We thank M. Mahé and N. Brynaert for preparing the tensile specimens and performing the tensile experiments. We greatly appreciate the advice of M. Bornert and E. Héripré regarding the DIC experiment. We thank J. H. Driver for improving the quality of the manuscript.

\section{Funding information}

Funding for this research was provided by: Agence Nationale de la Recherche (award No. ANR-11-BS09-030); European Synchrotron Radiation Facility (award No. HC/913).

\section{References}

Abu Al-Rub, R. K. \& Voyiadjis, G. Z. (2004). Int. J. Plast. 20, 11391182.

Boehler, J. P. \& Sawezuk, A. (1977). Acta Mech. 27, 185-206.

Bouscaud, D., Morawiec, A., Pesci, R., Berveiller, S. \& Patoor, E. (2014). J. Appl. Cryst. 47, 1699-1707. 
Chow, W., Solas, D., Puel, G., Baudin, T. \& Aubin, V. (2016). J. Mater. Sci. 51, 1234-1250.

Chung, J. S. \& Ice, G. E. (1999). J. Appl. Phys. 86, 5249-5255.

Dakhlaoui, R., Baczmański, A., Braham, C., Wroński, S., Wierzbanowski, K. \& Oliver, E. C. (2006). Acta Mater. 54, 5027-5039.

Demir, E., Raabe, D. \& Roters, F. (2010). Acta Mater. 58, 18761886.

Djaziri, S., Faurie, D., Renault, P. O., Le Bourhis, E., Goudeau, P., Geandier, G. \& Thiaudière, D. (2013). Acta Mater. 61, 5067-5077.

Eberl, F. (1999). PhD thesis, Ecole Nationale Supérieure d'Arts et Métiers - ENSAM, Paris, France.

El Shawish, S. \& Cizelj, L. (2016). Comput. Mater. Sci. 113, 133142.

Gouldstone, A., Chollacoop, N., Dao, M., Li, J., Minor, A. M. \& Shen, Y. L. (2007). Acta Mater. 55, 4015-4039.

Gravier, S., Coulombier, M., Safi, A., Andre, N., Boe, A., Raskin, J. P. \& Pardoen, T. (2009). J. Microelectromech. Syst. 18, 555-569.

Guery, A., Hild, F., Latourte, F. \& Roux, S. (2016). Mech. Mater. 100, 55-71.

Guery, A., Latourte, F., Hild, F. \& Roux, S. (2013). Meas. Sci. Technol. 25, 015401.

Guilhem, Y., Basseville, S., Curtit, F., Stéphan, J. M. \& Cailletaud, G. (2010). Int. J. Fatigue, 32, 1748-1763.

Héripré, E., Dexet, M., Crépin, J., Gélébart, L., Roos, A., Bornert, M. \& Caldemaison, D. (2007). Int. J. Plast. 23, 1512-1539.

Hirsch, P. B., Howie, A., Nicholson, R. B., Pashley, D. W. \& Whelan, M. J. (1965). Electron Microscopy of Thin Crystals, p. 496. London: Butterworths.

Hoc, T., Crépin, J., Gélébart, L. \& Zaoui, A. (2003). Acta Mater. 51, 5477-5488.

Hofmann, F., Eve, S., Belnoue, J., Micha, J. S. \& Korsunsky, A. M. (2011). Nucl. Instrum. Methods Phys. Res. Sect. A, 660, 130-137.

Imrich, P. J., Kirchlechner, C., Motz, C. \& Dehm, G. (2014). Acta Mater. 73, 240-250.

Johnson, G., King, A., Honnicke, M. G., Marrow, J. \& Ludwig, W. (2008). J. Appl. Cryst. 41, 310-318.

Kiener, D., Grosinger, W., Dehm, G. \& Pippan, R. (2008). Acta Mater. 56, 580-592.

Knauss, W. G., Chasiotis, I. \& Huang, Y. (2003). Mech. Mater. 35, $217-$ 231.

Larson, B. C., Yang, W., Ice, G. E., Budai, J. D. \& Tischler, J. Z. (2002). Nature, 415, 887-890.

Ledbetter, H. M. (1981). British J. Nondestr. Test. 23, 286-287.

Levine, L. E., Larson, B. C., Yang, W., Kassner, M. E., Tischler, J. Z., Delos-Reyes, M. A., Fields, R. J. \& Liu, W. (2006). Nat. Mater. 5, 619-622.

Lim, H., Carroll, J. D., Battaile, C. C., Boyce, B. L. \& Weinberger, C. R. (2015). Int. J. Mech. Sci. 92, 98-108.

Ludwig, W., Schmidt, S., Lauridsen, E. M. \& Poulsen, H. F. (2008). J. Appl. Cryst. 41, 302-309.
Marijon, J.-B. (2017). PhD thesis, Ecole Nationale Supérieure d'Arts et Métiers - ENSAM, Paris, France.

Mimura, H., Handa, S., Kimura, T., Yumoto, H., Yamakawa, D., Yokoyama, H., Matsuyama, S., Inagaki, K., Yamamura, K., Sano, Y., Tamasaku, K., Nishino, Y., Yabashi, M., Ishikawa, T. \& Yamauchi, K. (2010). Nat. Phys. 6, 122-125.

Monnet, G. \& Pouchon, M. A. (2013). Mater. Lett. 98, 128-130.

Petit, J., Castelnau, O., Bornert, M., Zhang, F. G., Hofmann, F., Korsunsky, A. M., Faurie, D., Le Bourlot, C., Micha, J. S., Robach, O. \& Ulrich, O. (2015). J. Synchrotron Rad. 22, 980-994.

Plancher, E. (2015). PhD thesis, Ecole Nationale Supérieure d'Arts et Métiers - ENSAM, Paris, France. https://pastel.archives-ouvertes.fr/tel-01304771.

Plancher, E., Petit, J., Maurice, C., Favier, V., Saintoyant, L., Loisnard, D., Rupin, N., Marijon, J. B., Ulrich, O., Bornert, M., Micha, J. S., Robach, O. \& Castelnau, O. (2016). Exp. Mech. 56, 483-492.

Pokharel, R., Lind, J., Li, S. F., Kenesei, P., Lebensohn, R. A., Suter, R. M. \& Rollett, A. D. (2015). Int. J. Plast. 67, 217-234.

Poulsen, H. F. (2012). J. Appl. Cryst. 45, 1084-1097.

Robach, O., Kirchlechner, C., Micha, J.-S., Ulrich, O., Biquard, X. \& Geaymond, O. (2014). Strain and Dislocation Gradients from Diffraction, edited by R. Barabash \& G. Ice, pp. 156-204. London: Imperial College Press.

Robach, O., Micha, J.-S., Ulrich, O., Geaymond, O., Sicardy, O., Härtwig, J. \& Rieutord, F. (2013). Acta Cryst. A69, 164-170.

Robach, O., Micha, J.-S., Ulrich, O. \& Gergaud, P. (2011). J. Appl. Cryst. 44, 688-696.

Roters, F., Eisenlohr, P., Hantcherli, L., Tjahjanto, D. D., Bieler, T. R. \& Raabe, D. (2010). Acta Mater. 58, 1152-1211.

Suquet, P., Moulinec, H., Castelnau, O., Montagnat, M., Lahellec, N., Grennerat, F., Duval, P. \& Brenner, R. (2012). Proc. IUTAM, 3, 6478.

Sutton, M. A., Li, N., Joy, D. C., Reynolds, A. P. \& Li, X. (2007). Exp. Mech. 47, 775-787.

Sutton, M. A., Yan, J. H., Tiwari, V., Schreier, H. W. \& Orteu, J. J. (2008). Opt. Lasers Eng. 46, 746-757.

Tasan, C. C., Diehl, M., Yan, D., Zambaldi, C., Shanthraj, P., Roters, F. \& Raabe, D. (2014). Acta Mater. 81, 386-400.

Van Swygenhoven, H. \& Van Petegem, S. (2013). Mater. Charact. 78, 47-59.

Vendroux, G. \& Knauss, W. G. (1998a). Exp. Mech. 38, 18-23.

Vendroux, G. \& Knauss, W. G. (1988b). Exp. Mech. 38, 86-92.

Wang, H., Clausen, B., Capolungo, L., Beyerlein, I. J., Wang, J. \& Tomé, C. N. (2016). Int. J. Plast. 79, 275-292.

Zhang, F. G., Bornert, M., Petit, J. \& Castelnau, O. (2017). J. Synchrotron Rad. 23, https://doi.org/10.1107/S1600577517006622.

Zhang, F. G., Castelnau, O., Bornert, M., Petit, J., Marijon, J. B. \& Plancher, E. (2015). J. Appl. Cryst. 48, 1805-1817.

Zhang, T., Jiang, J., Shollock, B. A., Britton, T. B. \& Dunne, F. P. (2015). Mater. Sci. Eng. A, 641, 328-339. 